\title{
Informationsservices auf Augenhöhe - So können Bibliotheken den Forschungsprozess proaktiv unterstützen
}

\author{
Felix Lohmeier, Jens Mittelbach, Matti Stöhr
}

Preprint eines Beitrags zu: Handbuch Informationskompetenz. Zweite, vollständig überarbeitete $u$. erweiterte Auflage. Hrsg. von Wilfried Sühl-Strohmenger. Unter Mitarbeit von Martina Straub. Verlag de Gruyter Saur: Berlin; Boston 2016.

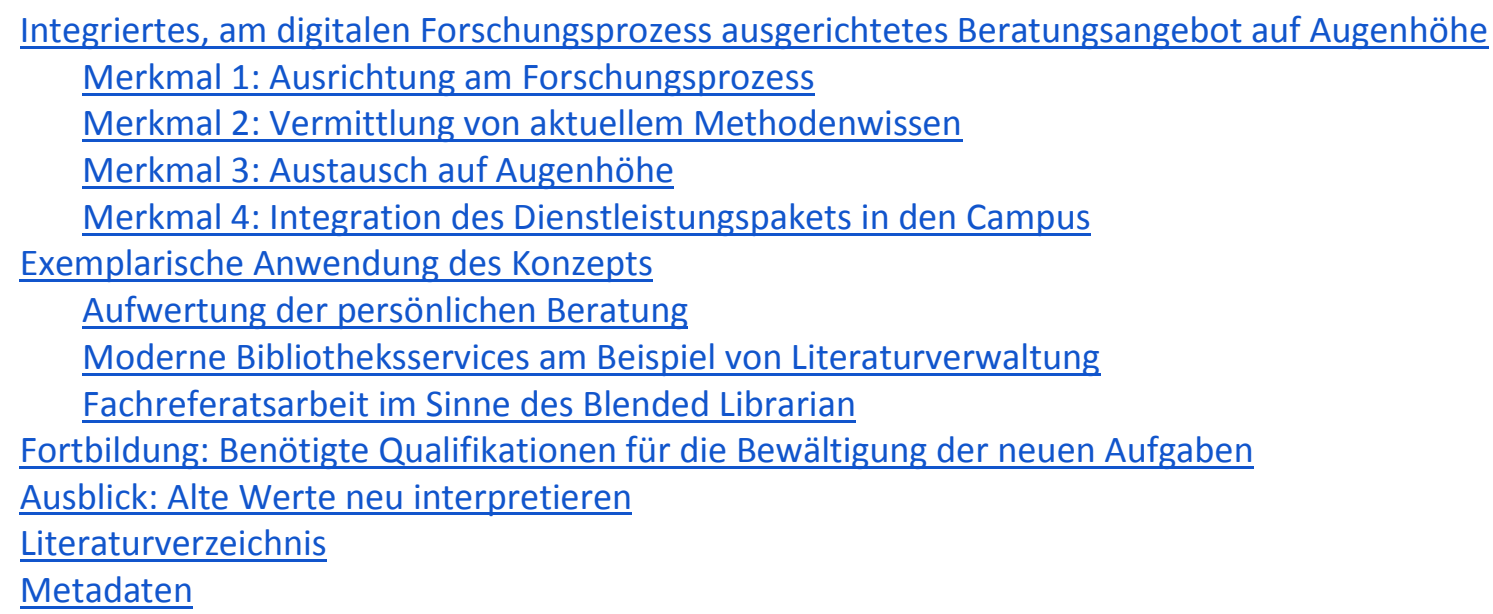

Es ist eine paradoxe Situation: Die durch Open Access und digitalisierte Forschung bedingte Transformation des wissenschaftsmethodischen Paradigmas erfordert ein besonders hohes Maß an Fähigkeiten und Fertigkeiten, die dem modernen Begriff von Informationskompetenz entsprechen. ${ }^{1}$ Gleichzeitig ist die im Wissenschaftssystem für Informationskompetenz eigens zuständige Einrichtung, die Bibliothek, immer weniger gefragt. Inhalte und didaktische Ansatzpunkte der bibliothekarischen Angebote zur Informationskompetenzvermittlung treffen offenbar nicht hinreichend die Bedürfnisse der Studierenden und Nachwuchswissenschaftlerlnnen. Wie können also Angebote der Bibliotheken und wissenschaftliche Nachfrage wieder in Einklang gebracht werden? In diesem Kapitel skizzieren wir ein Konzept für die Vermittlung von Informationskompetenz, das sich eng am (zunehmend digitalen) Forschungsprozess orientiert, exemplifizieren dieses am Beispiel von ausgewählten Bibliotheksangeboten und diskutieren die dafür notwendigen Qualifikationen. Die Überlegungen basieren auf praktischen Erfahrungen in der Erneuerung des Informationsservice an der SLUB Dresden in den Jahren 2012 bis 2014 sowie langjähriger Marktbeobachtung im Themenbereich Literaturverwaltung.

\section{Integriertes, am digitalen Forschungsprozess ausgerichtetes Bera- tungsangebot auf Augenhöhe}

Das Portal Informationskompetenz.de führt seit 2007 eine Veranstaltungsstatistik, ${ }^{2}$ zu der in den Jahren 2011 bis 2014 jeweils mindestens 73, überwiegend wissenschaftliche, Bibliotheken bei-

\footnotetext{
${ }^{1}$ Zur Begriffsdiskussion vgl. jüngst Hapke, Thomas: Was unter Informationskompetenz verstanden wird, verändert sich. In Hapke-Weblog (21.07.2015) http://blog.hapke.de/information-literacy/was-unterinformationskompetenz-verstanden-wird-veraendert-sich/ (7.8.2015) sowie den Beitrag

"Informationskompetenz anders denken - zum epistemologischen Kern von 'information literacy"” von Thomas Hapke in diesem Band. Preprint verfügbar unter http://dx.doi.org/10.15480/882.1249.

${ }^{2}$ Vgl. http://www.informationskompetenz.de/veranstaltungsstatistik/ (7.8.2015).
} 
getragen und pro Jahr mehr als 14.000 Veranstaltungen dokumentiert haben. Die inhaltlichen Schwerpunkte dieser Schulungsangebote haben sich in den letzten Jahren kaum verändert:

\begin{tabular}{|l|l|l|l|l|}
\hline Inhaltlicher Schwerpunkt (Mehrfachnennung möglich) & $\mathbf{2 0 1 1}$ & $\mathbf{2 0 1 2}$ & $\mathbf{2 0 1 3}$ & $\mathbf{2 0 1 4}$ \\
\hline Bibliotheksbenutzung & $66 \%$ & $63,48 \%$ & $63,39 \%$ & $63,72 \%$ \\
\hline Einzelne Kataloge und Datenbanken & $54 \%$ & $55,81 \%$ & $54,55 \%$ & $53,93 \%$ \\
\hline Suchstrategien und Suchtechniken & $41 \%$ & $38,4 \%$ & $40,88 \%$ & $39,69 \%$ \\
\hline Fernleihe/Dokumentlieferung & $23 \%$ & $22,74 \%$ & $20,39 \%$ & $25,17 \%$ \\
\hline$\ldots$ & & & & \\
\hline Elektronisches Publizieren & $1 \%$ & $1,1 \%$ & $1,29 \%$ & $1,39 \%$ \\
\hline
\end{tabular}

Auch im Jahr 2014 wird der Großteil der Schulungen durchgeführt, um die Benutzung von Diensten der Bibliothek zu erläutern, während - trotz aller Bemühungen um Open Access - der Anteil z.B. für elektronisches Publizieren verschwindend gering bleibt. Das muss sich ändern, wenn die wissenschaftlichen Bibliotheken auch in Zeiten der umfassenden Digitalisierung noch als ernstzunehmende Partner für die Forschung wahrgenommen werden wollen. Dabei bietet die digitale Transformation viele neue Tätigkeitsfelder, in denen bibliothekarische Expertise gefragt ist, darunter sind beispielsweise Forschungsdatenmanagement, Plagiatserkennung und -vermeidung, Literaturverwaltung, elektronisches Publizieren und Bibliometrie anzuführen. Wir plädieren für ein Beratungsangebot, dass sich

1. am Forschungsprozess ausrichtet,

2. aktuelles Methodenwissen vermittelt,

3. den Austausch auf Augenhöhe zum Ziel hat,

4. und sich als genuin bibliothekarisches Dienstleistungspaket in den Campus integriert.

Im Folgenden beschreiben wir diese vier konstitutiven Merkmale, bevor wir das Konzept an aktuellen Anwendungsbeispielen konkreter erläutern.

\section{Merkmal 1: Ausrichtung am Forschungsprozess}

Modellierungen des Forschungsprozesses können für Bibliotheken bei der Konzeptionierung ihres Service-Angebots und der Adressierung ihrer Zielgruppen sehr hilfreich sein. Ziel ist es, bibliothekarische Dienstleistungen bedarfs- bzw. kundenorientiert zu gestalten, also die Bibliothek als Produkt, dessen Bedienung erlernt werden muss, aus dem Fokus zu nehmen und dorthin die Kundlnnen ${ }^{3}$ mit ihrer Lebens- bzw. Arbeitswirklichkeit zu stellen. ${ }^{4}$ Um die Wirklichkeit einer Wissenschaftlerin, eines Magisterstudenten, einer Familienforscherin näherungsweise zu fassen, gehen wir pragmatisch von sechs Phasen des Forschungsprozesses aus:

1. Ideation, die Phase der Informationsrecherche, der Ideenfindung, der Themenidentifikation und -wahl;

2. Konzeptualisierung, die Phase der Methodenreflexion;

3. Operationalisierung, die Phase der Projektion der Methoden auf existente Infrastrukturen;

4. Kreation und Kollektion, die Phase des Experimentierens, Datenerzeugens und -sammelns;

5. Interpretation, die Phase der Datenauswertung und des wissenschaftlichen Schreibens, und

6. Dissemination, die Phase der Publikation der Forschungsergebnisse.

${ }^{3}$ Der Kundenbegriff ist in der Fachdiskussion höchst umstritten. Wir verwenden den Begriff, weil wir die Bibliotheken als Informationsdienstleister fokussieren.

${ }^{4}$ Vgl. hierzu den Beitrag Informationskompetenz als Kuratieren von Wissensräumen von Thorsten Bocklage, Julia Rübenstahl und Renke Siems in diesem Band. Preprint verfügbar via Researchgate:

https://www.researchgate.net/publication/278686492 Informationskompetenz als Kuratieren von Wisse nsrumen. 
Es geht uns dabei weniger um die exakte Beschreibung der tatsächlichen Schritte im Prozess des wissenschaftlichen Arbeitens, als vielmehr darum, an den generisch verstandenen Phasen des Forschungsprozesses relevante bibliothekarische Dienstleistungsangebote anzusiedeln. Gleichzeitig soll diese Abstraktion dazu dienen, den Forschungsprozess selbst besser zu reflektieren und damit Metawissen ${ }^{5}$ im Sinne des modernen Informationskompetenzbegriffes zu erzeugen. Auf diese Weise kann bei verschiedenen Zielgruppen latenter Bedarf hinsichtlich des Aufbaus von Kompetenzen manifest werden und Bemühungen auslösen, erkannte Lücken zu decken. Die an die Prozessphasen anzulagernden Dienstleistungen stellen wir uns dementsprechend - hier vereinfacht dargestellt ${ }^{6}-$ wie folgt vor:

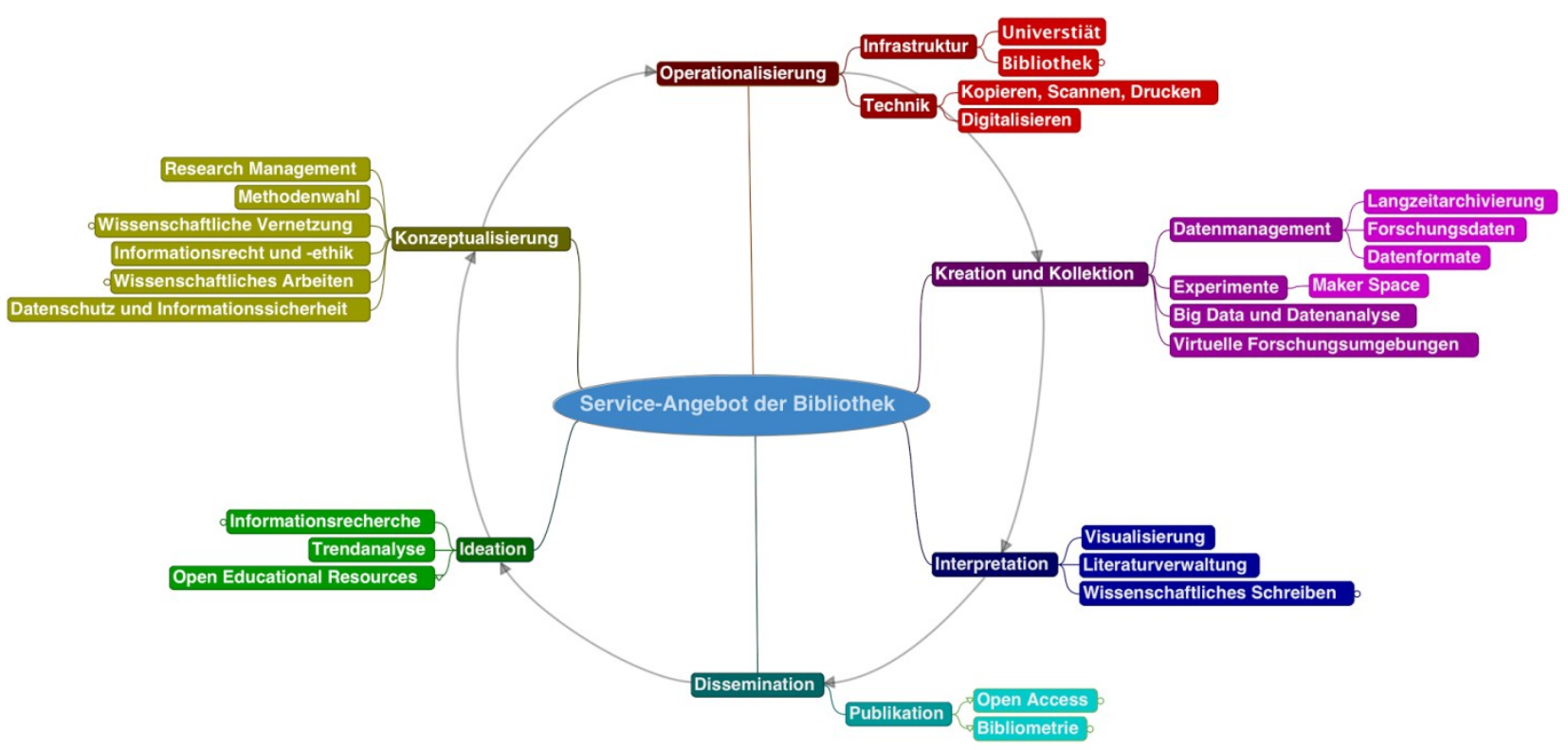

Abb. 1: Service-Angebot der Bibliothek, ausgerichtet an sechs Phasen des Forschungsprozesses

\section{Merkmal 2: Vermittlung von aktuellem Methodenwissen}

Zu den oben beschriebenen Phasen können Bibliotheken mehr beitragen als die bislang meistens üblichen Hilfestellungen zur Literaturrecherche. Wenn wir den ganzen Forschungsprozess betrachten, gibt es weitere Ansatzpunkte im Bereich der Methoden und Werkzeuge. Das lässt sich gut am Beispiel der Literaturverwaltung erläutern. Die Tätigkeit der Literaturverwaltung ist ein zentrales Mittel zur Begleitung von literaturgestützten Erkenntnis- bzw. Wissensprozessen und stellt dabei eine essentielle Handlung im wissenschaftlichen Arbeiten einschließlich Publizieren dar. ${ }^{7}$ Wie unter anderem Matthias Nagelschmidt 2010 und Thomas Stöber 2012 (in seinem Beitrag in der ersten Auflage dieses Handbuchs) nachvollziehbar feststellten, ist die Literaturverwaltung nicht isoliert zu betrachten. Sie ist eine selbstverständliche Teilaufgabe im „Kontext der Informationszirku-

\footnotetext{
${ }^{5}$ Vgl. Mackey, Thomas P. u. Trudi E. Jacobson: Metaliteracy. Reinventing information literacy to empower learners. Chicago: ALA Neal-Schuman 2014.

${ }^{6}$ Eine ausführlichere, interaktive und laufend weiterentwickelte Variante dieses Schemas finden Sie online unter http://www.jensmittelbach.de/service.html (10.08.2015).

${ }^{7}$ Vgl. Stöhr, Matti: Bibliothekarische Dienstleistungen für Literaturverwaltung. Eine vergleichende Analyse des Angebots wissenschaftlicher Bibliotheken unter besonderer Berücksichtigung der Nutzerperspektive. Magisterarbeit. Berlin 2010. S. 14-16. http://nbn-resolving.de/urn:nbn:de:kobv:11-100178393 (07.08.2015)
} 
lation" in engem Zusammenhang mit der Literaturrecherche, -beschaffung, -rezeption sowie anderen Teilschritten der Literaturarbeit: ${ }^{8}$

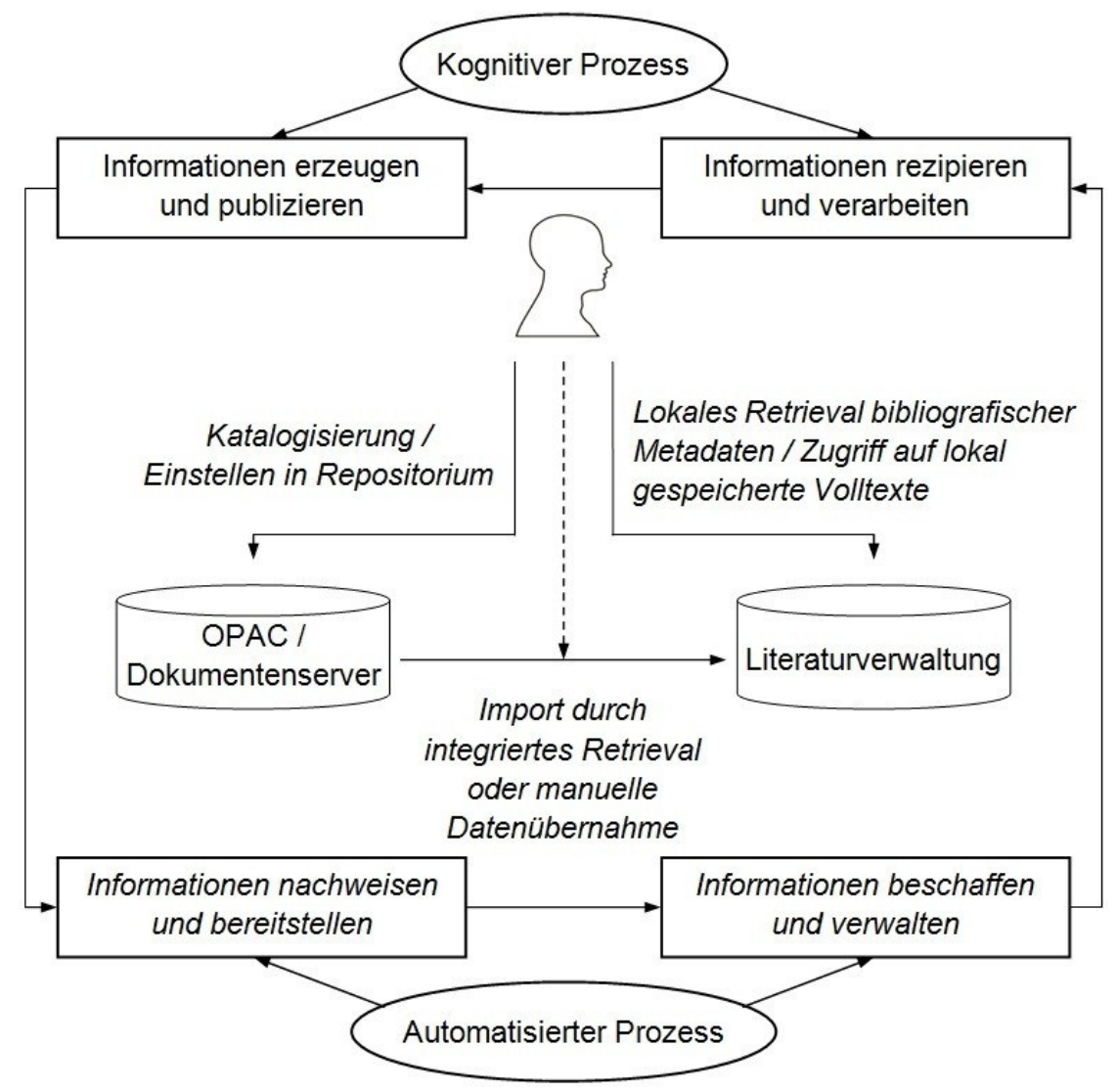

Abb. 2: „Literaturverwaltung im Kontext der Informationszirkulation“ (Nagelschmidt: Literaturverwaltungsprogramme, 2010. S. 96)

Literaturverwaltungsprogramme wurden in den letzten Jahren als Instrumente (weiter-)entwickelt, die den gesamten wissenschaftlichen Arbeits- bzw. Forschungsprozess von der Ideenfindung bis hin zur Manuskripterstellung und Publikation unterstützen. Folglich sind solche Programme treffender als "Research Management"-Tools beschrieben, da sie weit mehr bieten als "klassische Literaturverwaltung" - den strukturierten Import, die flexible Organisation und zitierstilgerecht-formatierten Export von Literaturnachweisen.

Bei der Vielfalt von Software-Varianten, funktionalen Möglichkeiten und unterschiedlichen Geschäftsmodellen kommt ein Unterstützungsbedarf bei (potentiellen) AnwenderInnen nicht von ungefähr. Sie wollen primär die für sie richtige Programmauswahl treffen und die Software schließlich für sich und ihre Anwendungsfälle effektiv einsetzen. Thomas Stöber hat unlängst deutlich gemacht:

Die Verwendung der Software ist sinnlos ohne die entsprechende Informations- und ITKompetenz, oder anders formuliert: die Literaturverwaltungssoftware ist das Tool - Informationskompetenz ist die Fähigkeit, dieses Tool gewinnbringend für die eigene wissenschaftliche

\footnotetext{
${ }^{8}$ Vgl. Nagelschmidt, Matthias: Literaturverwaltungsprogramme. Das funktionale Minimum. In: ABI-Technik 30 (2010) H. 2. S. 96. http://dx.doi.org/10.1515/ABITECH.2010.30.2.94 (07.08.2015) sowie Stöber, Thomas: Informationskompetenz und Literaturverwaltung. In: Handbuch Informationskompetenz. Hrsg. von Wilfried Sühl-Strohmenger. 1. Aufl. Berlin [u.a.]: De Gruyter 2012. S. 286. http://dx.doi.org/10.1515/9783110255188.281 (07.08.2015)
} 
Arbeit einzusetzen. Literaturverwaltung ist und bleibt damit eine Kernaufgabe für die Vermittlung von Informationskompetenz. ${ }^{9}$

Insbesondere wissenschaftliche Bibliotheken bieten bereits häufig Einstiegshilfen, etwa mit dem Angebot von Softwarevergleichen, ${ }^{10}$ können dies aber noch um individuelle lösungsorientierte Services ergänzen. Ein Beratungsangebot sollte neben der Auswahl der Werkzeuge den (fachspezifischen) konkreten Forschungsprozess betrachten und passendes Methodenwissen vermitteln. Wie hier am Beispiel Literaturverwaltung und Research Management deutlich wird, sollten Bibliotheken den Bedarf der NutzerInnen nach aktuellem kontextbezogenem Methodenwissen noch stärker adressieren.

\section{Merkmal 3: Austausch auf Augenhöhe}

Welche Beratungsangebote Nutzerinnen und Nutzer wahrnehmen wollen (latenter Bedarf) und aktiv nachfragen (manifester Bedarf), unterscheidet sich von dem, was die Bibliothek für angebotswürdig und notwendig erachtet. Die bibliothekarische Entscheidung, welche Dienstleistungen angeboten werden sollen ist außerdem auch davon abhängig, ob sie überhaupt kompetent und qualitativ hochwertig angeboten werden können. Idealerweise werden die Interessen und Fähigkeiten der BibliothekarInnen mit den Interessen der NutzerInnen in Einklang gebracht, so dass sich die drei Bereiche decken und somit ein fokussiertes, bedarfsgerechtes Angebot entsteht.

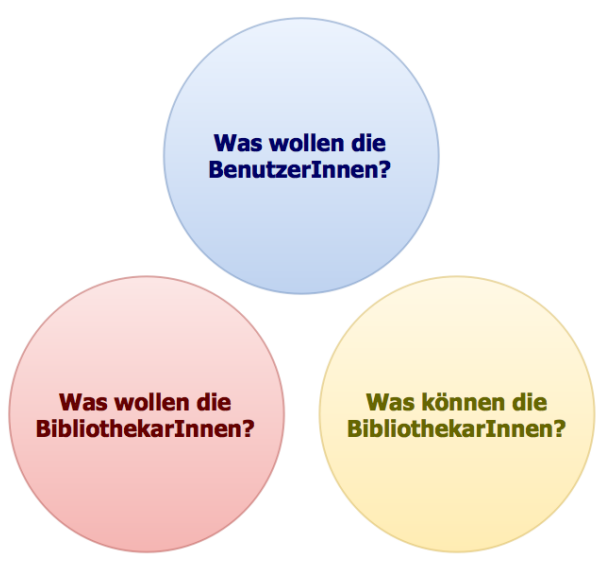

Unfokussiert
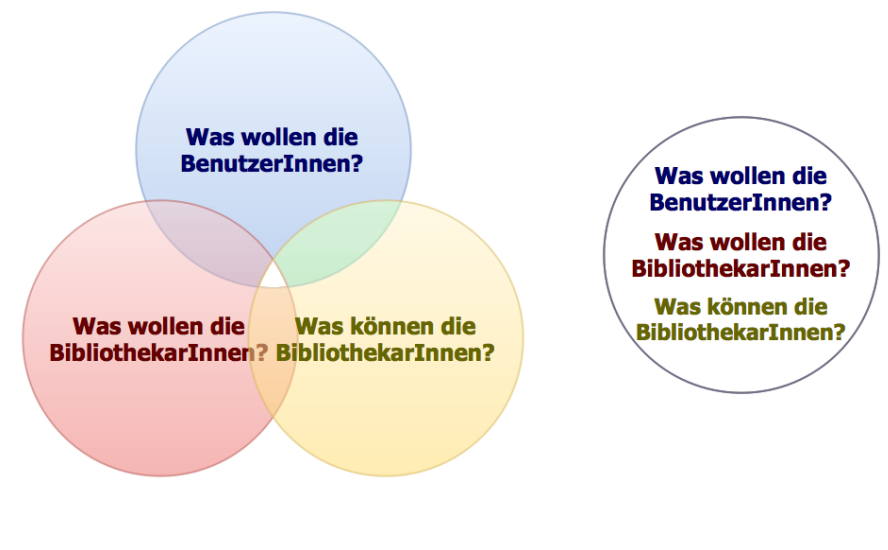

Fokussiert

Stark fokussiert

Abb. 3: Fokussierung des Service-Angebots

Es ist nicht nur Aufgabe der Bibliothekarinnen und Bibliothekare, auf manifesten Bedarf zu reagieren, sondern auch latenten Bedarf sicher zu identifizieren und den KundInnen bewusst zu machen. Gleichzeitig müssen sie die Dynamik akzeptieren, die der moderne Informationskompetenzbegriff impliziert und sich im Prozess des lebenslangen Lernens selbst ständig adäquates Methodenwissen aneignen. Beides ist nur denkbar, wenn die Interaktion mit den Zielgruppen auf Augenhöhe - also im Sinne des Konzeptes des Blended Librarian bzw. Embedded Librarian $^{11}$

\footnotetext{
${ }^{9}$ Stöber: Informationskompetenz. S. 285 (siehe Fn. 8).

${ }^{10}$ Als prägnante Beispiele seien Softwarevergleiche der UB TU München sowie der SLUB Dresden genannt: Lemke, Dorothea, Jana Votteler u.a.: Softwarevergleich Literaturverwaltung - 5. Aktualisierung 2015 (30.6.2015). https://mediatum.ub.tum.de/node?id=1271693 (7.8.2015); Musiat, Jutta, Annika-Valeska Walzel u. Christina Wenzel: Literaturverwaltungsprogramme im Überblick (Januar 2015). http://slubdd.de/5b (7.8.2015).

${ }^{11}$ Vgl. die aktualisierte Perspektive auf die bereits 2004 entwickelten Vorstellungen zu Blended Librarianship: Shank, John D., Steven Bell u. Diane Zabel: Blended librarianship. [Re]envisioning the role of librarian as
} 
stattfindet. Das bedeutet, die Kompetenzen der KundInnen einzubeziehen und authentische Kommunikationssituationen zu schaffen, in denen durchaus beide Seiten voneinander bzw. miteinander lernen. In der Auskunftspolicy einer Bibliothek, die so arbeiten möchte, könnte demzufolge dieser Paragraph zu finden sein:

Das Auskunfts- und Beratungsgespräch findet auf Augenhöhe statt. Wir Mitarbeiterlnnen vermeiden belehrende und zurechtweisende Kommunikationsstrategien. Die Kenntnisse der KundInnen beziehen wir aktiv in die Problemlösung ein. Aufgrund eines transparenten und schlüssigen Dialogs fühlen sich unsere KundInnen gut beraten. Ein Beratungsgespräch ist in unseren Augen besonders gelungen, wenn die KundInnen den Eindruck gewinnen, dass auch die Mitarbeiterin oder der Mitarbeiter dabei etwas gelernt hat.

\section{Merkmal 4: Integration des Dienstleistungspakets in den Campus}

Aus den zuvor beschriebenen Merkmalen ergibt sich bereits ein modernes bibliothekarisches Beratungsangebot, das seine Wirkung jedoch nur optimal entfalten kann, wenn es erstens in sich verschränkt ist und zweitens in den Campus integriert wird. Egal, an welcher Stelle die Nutzerin oder der Nutzer mit der Bibliothek in Kontakt tritt (ob an der Theke, in einer Spezialberatung oder via technische Dienste), es sollten immer individualisiert und kontextbezogen weitere passende Angebote aus dem Dienstleistungspaket offenbar werden. Zudem sollte die ganzheitliche Beratung nicht an den Türen der Bibliothek halt machen, sondern andere Angebote auf dem Campus einbeziehen. Dazu ist es notwendig das genuin Bibliothekarische des Angebots herauszuarbeiten und somit ein Dienstleistungsportfolio zu bestimmen, dass sich komplementär in den Campus integriert.

Dabei ist es wichtig zu beachten, dass die Erwartungshaltung an Bibliotheken fest in den Köpfen der NutzerInnen verankert ist. Cathy De Rosa et al. haben in einer Studie die "Campus Perception Map" ${ }^{\prime 2}$ entwickelt, die auf einer Onlinebefragung von US-amerikanischen Alumni und Eltern von Studierenden beruht. Die Probanden mussten u.a. für jeden Ort auf dem Campus angeben, wie stark sie diesen mit bestimmten Aktivitäten verbinden. Auf dieser Basis ist eine mentale Landkarte entstanden (vgl. Abbildung 4, Orte sind grün hervorgehoben, die im Zusammenhang mit bestimmten dort ausgeführten Aktivitäten stehen). Wenig überraschend wird die Bibliothek als ruhiger Ort für Einzelarbeit verstanden, an dem drei Aktivitäten besonders hohe Werte erhalten: "Get work done", "basic technology tools to get work done" und "providing current, relevant information". Wenn also die oben skizzierten Dienstleistungsangebote erfolgreich angenommen werden sollen, muss das Verständnis für den Ort "Bibliothek" weiterentwickelt werden. Forschungsprozess-relevante Dienstleistungen müssen aktiv beworben werden, weil die NutzerInnen nicht von allein auf die Idee kommen würden, sie in Bibliotheken zu suchen. Hilfreich kann hierbei die bewusste Neuprägung einer Corporate Identity sein, beispielsweise durch ein einheitliches Corporate Design, das im Zuge der neuen Angebote eingeführt wird. ${ }^{13}$

educator in the digital information age. In: Reference and User Services Quarterly 51 (2011) H. 2. S. 105110. http://www.jstor.org/stable/refuseserq.51.2.105 (7.8.2015). Dort auch Hinweise zur Abgrenzung von Embedded Librarianship und Librarianship 2.0.

${ }^{12}$ DeRosa, Cathy, Joanne Cantrell u.a.: At a tipping point. Education, learning \& libraries ; a report to the OCLC membership. Dublin, OH, USA: OCLC 2014. S, 62. https://www.oclc.org/content/dam/oclc/reports/tippingpoint/215133-tipping-point.pdf

${ }^{13}$ Vgl. zum Beispiel die Neuausrichtung der ZBW 2010, welche als Stütze der Veränderung gleichzeitig ein neues Corporate Design eingeführt hat: Siegfried, Doreen: Wissenschaftsservice der Zukunft. ZBW definiert Forschungssupport neu. Pressemitteilung der ZBW (7.12.2010). http://www.zbw.eu/de/ueberuns/aktuelles/meldung/news/wissenschaftsservice-der-zukunft-zbw-definiert-forschungssupport-neu/ (7.8.2015). 


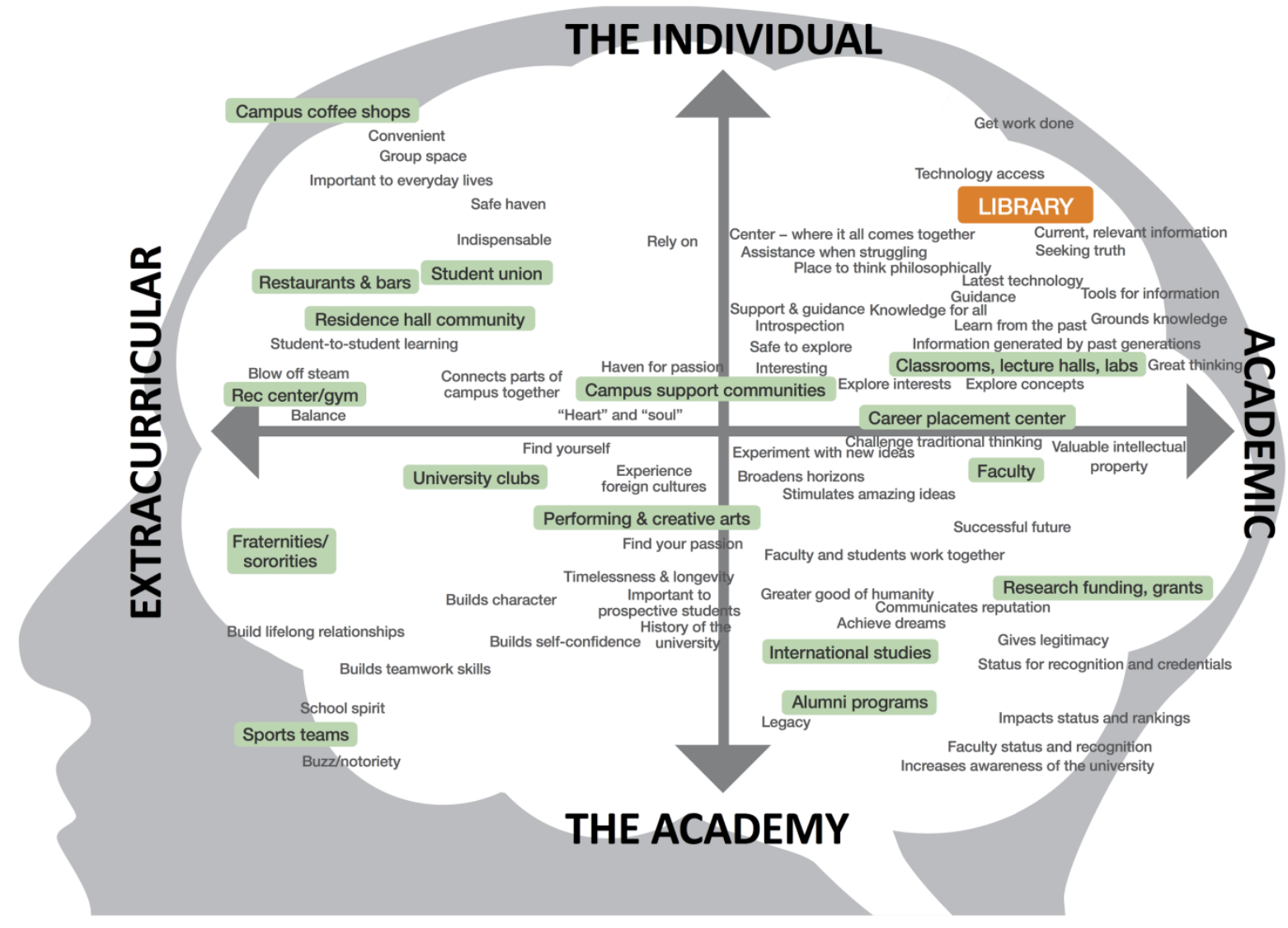

Abb. 4: „Campus Perception Map“ (De Rosa, Cantrell u.a: Tipping point, 2014. S. 62)

Für die Integration des Dienstleistungspakets in den Campus sollte unserer Auffassung nach ein komplementäres Angebot zu den Einführungskursen ins wissenschaftliche Arbeiten der Fachdisziplinen, der technischen Beratung am Rechenzentrum und den ggf. vorhanden Lehr- und Lernzentren entstehen, welche die Besonderheiten des jeweiligen Campus berücksichtigt und die Stärken und Kenntnisse der jeweiligen Bibliothek herausstellt. Gleichzeitig sind Bibliotheken auch ideale Orte, um andere zivilgesellschaftliche Akteure außerhalb des Campus (HobbyforscherInnen, AktivistInnen, SammlerInnen) einzubinden. Wie R. David Lankes 2012 überspitzt, aber treffend formulierte, sollten wir uns bemühen Communities an die Bibliotheken zu binden:

Bad Libraries build collections. Good libraries build services (of which a collection is only one). Great libraries build Communities. ${ }^{14}$

\section{Exemplarische Anwendung des Konzepts}

Was bedeutet das für die alltägliche Arbeit in Bibliotheken? Was muss sich konkret ändern? Um das hier vorgeschlagene integrierte Konzept für die Vermittlung von Informationskompetenz näher zu erläutern, wollen wir die Konsequenzen der oben skizzierten Merkmale am Beispiel von drei Bibliotheksdienstleistungen erläutern: Persönliche Beratungsangebote, Services für Literaturverwaltung und Fachreferatsarbeit.

\footnotetext{
${ }^{14}$ Lankes, R. David: Beyond the bullet points. Bad libraries build collections, good libraries build services, great libraries build communities (11.3.2012). http://quartz.syr.edu/blog/?p=1411 (7.8.2015).
} 


\section{Aufwertung der persönlichen Beratung}

Den Benutzerinnen und Benutzern wird bibliothekarische Beratung klassischerweise an Informationstheken, über einzelne fachliche AnsprechpartnerInnen oder auf schriftlichem Wege angeboten. Als Angebotsmatrix dienen meist die Organisationsstrukturen der Bibliothek, Wissen darüber wird oft ebenso stillschweigend vorausgesetzt wie die zumindest grobe Kenntnis bibliothekarischer Workflows. Da die Beantwortung spezifischer Fragen an den Auskunftstheken in der ad-hocSituation von der Zufälligkeit abhängt, mit der dort ein fachlich kompetenter Ansprechpartner angetroffen wird, beschränken sich NutzerInnen in vielen Fällen darauf, allgemeinere Fragen zu stellen. Für fachliche Anliegen müssen sie sich oft genug die richtige Person selbst heraussuchen. Das Resultat ist im schlimmsten Fall ein Teufelskreis aus sinkender Nachfrage, abnehmender Informationskompetenz auf bibliothekarischer Seite, Rückbau von Service-Strukturen und sinkender Metakompetenz auf Nutzerseite. Die Bibliothek büßt an einer Stelle ein, an der eigentliche ihre große Stärke liegen könnte.

Das aus dem angloamerikanischen Raum bekannte Konzept der "Research Consultations" setzt auf stärkere Orientierung an den tatsächlichen Bedürfnissen der BenutzerInnen. Eine Möglichkeit, BibliotheksnutzerInnen in den Genuss hochwertiger und spezifischer fachlicher Beratung kommen zu lassen, ist eine themenspezifische Aufbereitung des Beratungsangebotes. An größeren Bibliotheken sind oft bereits Ticketsysteme im Einsatz, die schriftliche Anfragen selbständig an die jeweils kompetenten AnsprechpartnerInnen verteilen. Die SLUB Dresden geht mit ihrem Service Wissensbar noch einen Schritt weiter und gibt ihren NutzerInnen, zusätzlich zu einem Basis-Beratungsangebot an den Theken, für spezifischere Fragestellungen Zugriff auf die Terminkalender der dafür passenden ExpertInnen. ${ }^{15}$ Dadurch kann die Bibliothek mehreren Prinzipien gerecht werden. Einerseits erreicht sie eine Verbesserung der Wahrnehmung des bibliothekarischen Service-Portfolios im Sinne der oben erwähnten "Campus Perception Map:" Der affektive Wert der bibliothekarischen Services steigt, weil sie in der Konzeptionierung von der Benutzerperspektive her als persönlich, "mit Gesicht" ausgestattet, auf Augenhöhe angeboten und damit als relevant erscheinen. Gleichzeitig wird die Informationskompetenz der KundInnen insbesondere im Bereich der Metakompetenzen gesteigert, weil in der Auseinandersetzung mit dem Portfolio der Themen in erster Linie die für die Forschung relevanten Tätigkeiten und Aufgabenstellungen transparent werden, und nicht mehr vornehmlich die bibliothekarischen Abläufe aufleuchten. Latent vorhandener Bedarf hinsichtlich des Ausbaus von Informations- bzw. Forschungskompetenz kann so den Benutzerinnen und Benutzern bewusst - und damit manifest - werden. Hervorzuheben ist auch der Ansporn für die Beraterlnnen, sich permanent fortzubilden - ein Ansporn, der aus deren nunmehr konkreten Verantwortlichkeit für spezifische Themen resultiert, die die bislang vorherrschende, mehr mehr oder weniger vage Fachgebietszuständigkeit ablöst bzw. ergänzt.

\section{Moderne Bibliotheksservices am Beispiel von Literaturverwaltung}

Bibliotheken und BibliothekarInnen sind aufgrund ihrer Kernaufgaben und -kompetenzen, nicht zuletzt aber aufgrund ihrer (Neu-)Definition als den wissenschaftlichen Arbeitsprozess aktiv begleitende und fördernde Informationsdienstleister, prädestiniert für die breite Unterstützung persönlicher softwarebasierter Literaturverwaltung und Wissensorganisation. Entsprechende Serviceangebote sind unlängst in die bibliothekarische Servicepraxis, vorrangig in wissenschaftlichen Bibliotheken mit Hochschulanbindung, integriert und werden sehr gut angenommen, wie einige

\footnotetext{
${ }^{15}$ Vgl. z.B. Mittelbach, Jens u. Antonie Muschalek: Wissensbar. Experten beraten passgenau. In: BuB: Forum Bibliothek und Information 67 (2015) H. 6. S. 374-376. http://b-u-b.de/wp-content/uploads/2015-06.pdf. Guercke, Olaf: Bequem erreichbar und maßgeschneidert, Persönliche Beratungsangebote in der Digitalen Bibliothek. Olaf Guercke und Jens Mittelbach im Gespräch über die Wissensbar der SLUB Dresden. Interview mit Jens Mittelbach (3.8.2015). Erschienen in: b. i. t. online 18 (2015), H. 3. S. 267-268. http://www.b-i-tonline.de/heft/2015-03-interview-guercke.pdf.
} 
bereits mehrere Jahre zurückliegende systematische Untersuchungen bereits gezeigt haben. ${ }^{16}$ So hat Thomas Stöber verdeutlicht:

Literaturverwaltung ist damit gewissermaßen eine Querschnittstechnik, die eine integrierte [Dienstleistungs-]Perspektive geradezu fordert. Wenn eine solche integrierte Perspektive angestrebt ist, ist zugleich jedoch eine enge Abstimmung zwischen Fachbereich und Bibliothek notwendig; im Idealfall greifen die wissenschaftspropädeutischen Angebote von Fachbereich und Bibliothek ineinander. ${ }^{17}$

Das ist nur konsequent, geht man mit Stöber davon aus, dass sich Alltag und Routinen wissenschaftlicher Arbeitswirklichkeit zum "digitalen Schreibtisch" entwickeln. ${ }^{18}$ Dies berücksichtigend, lässt sich das Dienstleistungsspektrum grundsätzlich in vier Kategorien typologisch beschreiben: ${ }^{19}$

- Informierende Dienstleistungen: Was heißt systematische Literaturverwaltung? Welche Hilfsmittel gibt es dafür? Wie kann ich diese bewerten und schließlich das für mich geeignete Produkt auswählen? Hier geht es um die Vermittlung von theoretischen Grundlagen der systematischen softwaregestützten Literaturverwaltung bzw. des Research Managements als Tätigkeit und um die Verdeutlichung von Mehrwerten durch die systematische Softwarenutzung. Dazu gehört die Einordnung eines campuslizenzierten oder anderweitig zur Unterstützung ausgewählten Programms in den Softwaremarkt, meist in Vergleichsform, mit Überblick von Vor- und Nachteilen.

- Best Practice-Beispiele: "Entscheidungsbaum" der UB Konstanz; Umfassender Softwarevergleich der UB TU München; Adaptiver Softwarevergleich der Zürcher Hochschule für Angewandte Wissenschaften (ZHAW) - Hochschulbibliothek ${ }^{20}$

- Programmbezogen-unterstützende Dienstleistungen: Bibliotheken ermöglichen einen niederschwelligen Softwareerwerb, etwa über den Abschluss von Campuslizenzen. Gleichzeitig unterstützen Sie vor allem EinsteigerInnen aber auch Fortgeschrittene mit Informationsmaterial (Hilfe zur Selbsthilfe) und vielgestaltigen Schulungen im versierten Umgang mit - in der Regel - dem bzw. den lizenzierten Produkt(en); (kostenfreie) Alternativen werden zielgrup-

${ }^{16}$ Süselbeck, Kirsten: Literaturverwaltung als Teil des Konzepts zur Vermittlung von Informationskompetenz an wissenschaftlichen Bibliotheken 2011. http://bvbm1.bib-bvb.de/publish/viewer/16/2805073.html (7.8.2015); Stöber, Thomas: Serviceangebote der wissenschaftlichen Bibliotheken im Bereich Literaturverwaltung: Ergebnisse einer Umfrage. Augsburg 2010. http://opus.bibliothek.uniaugsburg.de/volltexte/2010/1611 (7.8.2015) und Stöhr: Bibliothekarische Dienstleistungen, 2010. Auf der Basis der Ergebnisse entstand die im Kontext der Webplattform “Literaturverwaltung" mit dem Dienst Google Maps kontinuierlich gepflegte Übersicht "Services für Literaturverwaltung - die Karte”, vgl. https://literaturverwaltung.wordpress.com/ansprechpartner/ (7.8.2015). Vgl. auf internationaler Ebene: Francese, Enrico: Reference management software as digital libraries. A survey at the University of Torino. Master Thesis. Oslo 2012. http://hdl.handle.net/10642/1274 (7.8.2015) und McMinn, Howard Stephen: Library support of bibliographic management tools. A review. In: Reference Services Review 39 (2011) H. 2. S. 278-302. http://dx.doi.org/10.1108/00907321111135493 (7.8.2015)

${ }^{17}$ Stöber: Informationskompetenz. S. 286 (siehe Fn. 8).

${ }^{18}$ Vgl. ebd., S. 286-288 und zuletzt Stöber, Thomas: Der "digitale Schreibtisch". Innovationsmanagement und Vernetzung für das wissenschaftliche Arbeiten. In: Literaturwissenschaft im digitalen Medienwandel. Hrsg. von Christof Schöch u. Lars Schneider. Berlin 2014 (PhiN. Philologie im Netz: Beihefte). S. 180-188. http://userpage.fu-berlin.de/ phin/beiheft7/b7t11.pdf (7.8.2015).

${ }^{19}$ Vgl. ausführlich Stöhr: Bibliothekarische Dienstleistungen, 2010. S. 63-85 (siehe Fn. 16).

${ }^{20}$ Vgl. jew. http://www.ub.uni-konstanz.de/serviceangebote/literaturverwaltung.html; http://mediatum.ub.tum.de/node?id=1127579; http://www.zhaw.ch/de/zhaw/hochschulbibliothek/dienstleistungen/literaturverwaltung.html (7.8.2015). 
pengerecht einbezogen. Direkte Kontaktservices durch sichtbare Ansprechpersonen, etwa in Form von verbindlichen Sprechstundenzeiten für individuelle Beratung, runden das Dienstleistungssegment ab.

- Best Practice-Beispiele: frei nutzbare Citavi-Schulungsmaterialien der SUB Göttingen, Wissensbar der SLUB Dresden; fachbezogene Ansprechpersonen der Bodleian Libraries Oxford ${ }^{21}$

- Datenbezogen-unterstützende Dienstleistungen: Auf welchen Wegen können bibliographische Daten als Einzeltreffer oder Ergebnislisten aus dem klassischen Bibliotheks-OPAC und/oder falls angeboten - aus dem integrierten Discovery-System nachgenutzt werden? Welche (lizenzierten) Datenbanken können in welcher Form ebenso berücksichtigt werden? Entsprechende katalog- bzw. systemtechnische Konfigurationen und Funktionsfreigaben sind die Basis für die Datenübernahme in persönliche Literaturverwaltungen. Unterstützung im korrekten Zitieren im Kontext der Sicherung guter wissenschaftlichen Praxis u.a. in der Bereitstellung von Zitierstilen sind hier ebenso aufzuführen.

- Best-Practice-Beispiele: FAQ zu Literaturverwaltungsschnittstellen an der UB Bielefeld; Import/Export-Wiki für Literaturverwaltungsprogramme der UB Konstanz; Tutorial LOTSE z.B. an der SUB Hamburg ${ }^{22}$

Kontexterweiternde Dienstleistungen: Welche Möglichkeiten gibt es die in der eigenen oder kollaborativ genutzten Literaturverwaltungsumgebung organisierten Daten (und Volltexte) möglichst verlustfrei in andere, im Kontext der Forschung und Lehre, zu verwendende Informationssysteme zu übertragen und anwendungsbezogen nachzunutzen?

- Best-Practice-Beispiele: Forschungsinformationssystem PUMA an der Universität Kassel; Hochschulbibliographien an den Universitäten Bochum, Dortmund und Duisburg-Essen ${ }^{23}$

Am Beispiel der zahlreichen Möglichkeiten im Bereich Literaturverwaltung zeigt sich, wie aus einzelnen Bausteinen ein integriertes, am digitalen Forschungsprozess ausgerichtetes Beratungsangebot entstehen kann. Laufende Fachinformationen und Austauschmöglichkeiten zum Thema finden sich u.a. auf der Webplattform "Literaturverwaltung", vormals "Literaturverwaltung \& Bibliotheken". ${ }^{24}$

${ }^{21}$ Vgl. jew. http://www.slub-dresden.de/service/wissensbar/thema/gbList/10/; http://ox.libguides.com/content. php?pid=294548\&sid=2418232 (7.8.2015).

${ }^{22}$ Vgl. jew. http://www.ub.uni-bielefeld.de/library/literaturverwaltung/schnittstellen.htm; https://ilias.unikonstanz.de/ilias/goto.php?target=wiki 202449; https://lotse.sub.unihamburg.de/fachuebergreifend/arbeiten schreiben und veroeffentlichen/literatur zitieren/literatur zitier en-de.php (7.8.2015).

${ }^{23}$ Vgl. jew. http://www.academic-puma.de, http://bibliographie-trac.ub.rub.de (7.8.2015).

${ }^{24}$ Stöhr, Matti: Neuer Titel, neue Möglichkeiten - “Literaturverwaltung \& Bibliotheken” heißt nun "Literaturverwaltung". In: Literaturverwaltung (10.05.2013).

https://literaturverwaltung.wordpress.com/2013/05/10/neuer-titel-neue-moglichkeitenliteraturverwaltung-bibliotheken-heist-nun-literaturverwaltung/ (7.8.2015). Zum Weblog als Kernstück und der bereits erwähnten Servicekarte gehören auch eine Verbindung zur deutschen Informationskompetenzplattform http://www.informationskompetenz.de/im-fokus/literaturverwaltung/ (7.8.2015), "Reference Management"-Gruppen in Zotero und Mendeley zwecks bibliographischem Publikationsnachweis zum Thema (vgl. https://literaturverwaltung.wordpress.com/bibliographie/), sowie die Facebook-Seite "Literaturverwaltung im Fokus" (https://www.facebook.com/Literaturverwaltung) und der Twitterkanal "Literaturverwaltung" (https://twitter.com/Litverwaltung) (7.8.2015). 


\section{Fachreferatsarbeit im Sinne des Blended Librarian}

Die Aufgabenschwerpunkte im Fachreferat haben sich von Erwerbung und Erschließung eindeutig hin zu mehr Vermittlung verschoben, um ein einheitliches Aufgabenprofil wird jedoch immer noch gestritten. ${ }^{25}$ Da strukturelle Änderungen auf sich warten lassen, sind die Individuen gefragt, das Konzept des "Blended Librarian" mit Leben zu füllen. Was also können FachreferentInnen tun, wenn sie den oben skizzierten Merkmalen eines integrierten Beratungsangebots folgen wollen?

Zunächst sollten sie gegenwärtige Werkzeuge und Arbeitsweisen derjenigen Fachcommunities genau kennenlernen und verstehen, für die sie zuständig sind. Angesichts der hohen Entwicklungsdynamik der wissenschaftlichen Methoden ist das nicht trivial und erfordert ständige Fortbildung und direkten Kontakt mit den WissenschaftlerInnen. Auf Basis eines gegenwärtigen Methodenwissens des jeweiligen Fachs könnten FachreferentInnen dann beurteilen, welche der stetig neu entstehenden Tools $^{26}$ für methodische Innovationen tauglich sind und an welcher Stelle die Bibliothek die Forschung konkret unterstützen kann. Wer die fachspezifischen Anforderungen kennt und sich breites Wissen über neue wissenschaftliche Werkzeuge verschafft, kann den interdisziplinären Austausch unterstützen und zwischen Generellem und Spezifischem vermitteln.

Erfolgversprechend ist es zudem, die Beratungsangebote an technische Dienstleistungen der Bibliothek zu koppeln. So entstehen beispielsweise im Zuge der Ablieferung einer elektronischen Publikation auf einem Open-Access-Server häufig verwandte Fragen zur Publikation von zugrundeliegenden Forschungsdaten, zu Lizenzfragen oder zu bibliometrischen Auswertungen. Daraus können integrierte umfassende Serviceleistungen wie eine Open-Access-Rechteprüfung inkl. Publikation ${ }^{27}$ oder einer Beratung zu Forschungsdatenmanagement inkl. technischen Lösungsangeboten ${ }^{28}$ führen. Naheliegend ist auch eine fachbezogene Unterstützung bei der Schaffung bedarfsgerechter Literaturverwaltungs- und Research-Management-Umgebungen, wie im vorigen Abschnitt beschrieben. Diese Bedarfe zu identifizieren, Angebote zu konzeptionieren und diese in Forschung und Curricula zu integrieren, ist und bleibt eine originäre Aufgabe der FachreferentInnen.

\section{Fortbildung: Benötigte Qualifikationen für die Bewältigung der neuen Aufgaben}

Der Paradigmenwechsel im Verständnis des Begriffes Informationskompetenz fordert von den BibliothekarInnen als scholars' scholars ${ }^{29}$ zuallererst selbst Informationskompetenz auf- und auszubauen. Längst herausgearbeitet sind in der Literatur Konzepte des lebenslangen Lernens, für die neben dem formellen Wissenserwerb in unserem digitalisierten Ökosystem informelles Lernen

\footnotetext{
${ }^{25}$ Vgl. aktuelle Kurzzusammenfassung bei Tappenbeck, Inka: Fachreferat 2020. ZBW Hamburg 2014 (VDB: Fachreferententagung Wirtschaftswissenschaften). http://www.fbi.fhkoeln.de/institut/personen/tappenbeck/publikationen/Fachreferat 2020 Praesentation.pdf (7.8.2015).

${ }^{26}$ Ein guter Einstieg ist die folgende von der Universitätsbibliothek Utrecht herausgegebene Übersicht: Bosman, Jeroen u. Bianca Kramer: 101 Innovations in Scholarly Communication. http://innoscholcomm.silk.co (7.8.2015).

${ }^{27}$ Vgl. Di Rosa, Elena u. Michaela Voigt: Qualitätssicherung für Serviceangebote zur Rechteprüfung für Bibliotheken. Bremen 2014 (103. Deutscher Bibliothekartag). http://nbnresolving.de/urn/resolver.pl?urn:nbn:de:0290-opus-16149 (8.7.2015).

${ }^{28}$ Vgl. Berstler, Susan, Christopher Erdmann u.a.: Data Management at Harvard. http://isites.harvard.edu/icb/icb.do?keyword=k78759 (7.8.2015).

${ }^{29}$ Vgl. Levitin, Daniel J.: The organized mind. Thinking straight in the age of information overload. New York, NY: Dutton 2014. S. 339.
} 
eine immer größere Rolle spielt. ${ }^{30}$ Vor allem gilt es, sich bewusst den Herausforderungen zu stellen, ja sie geradezu zu suchen, indem man eben solche Pfade beschreitet, auf denen man sich am wenigsten sicher fühlt. ${ }^{31}$

In Institutionen setzt sich die Einsicht durch, dass sie zusammen mit ihren Mitarbeiterinnen und Mitarbeitern vor einer großen Herausforderung stehen; der Ruf, sich fortzubilden, ist für jede Biblibliothekarin, für jeden Bibliothekar unüberhörbar geworden. Gleichzeitig wird immer noch zu wenig und oft genug nicht das Richtige für die professionelle Fortbildung getan. Die hier vorgeschlagene Fokussierung auf den Forschungsprozess bedeutet die Hinwendung zu den Belangen der BenutzerInnen von Bibliotheken. Unsere Fortbildung muss sich daher von ihrem bibliothekarischen Solipsismus befreien und sich die Herausbildung von Informationskompetenz im erweiterten Sinne zum Ziel setzen. Um die individuelle auf die Unterstützung des Forschungsprozess ausgerichtete Fortbildung Ihrer MitarbeiterInnen zu unterstützen, sollte die Institution einen verbindlichen, partizipativen Rahmen schaffen, beispielsweise in Form von Kolloquien, Workshops usw., orchestriert und begleitet von hochengagierten und kompetenten Fortbildungsbeauftragten. In Fortbildung investierte Arbeitszeit muss als gut investierte Zeit anerkannt werden. Experimentelles Lernen, ${ }^{32}$ die eigenverantwortliche Rezeption informeller Kanäle, ${ }^{33}$ das Verfolgen von Blogs ${ }^{34}$ und eigenes Bloggen, das gemeinsame Schreiben mit KollegInnen, ${ }^{35}$ das gemeinsame Lernen mit den BenutzerInnen können so neben die systematischen Lernformen treten. In solch einer auch auf das bibliothekarische Backoffice ausgeweiteten Atmosphäre des Lernens nimmt das Diktum von der Bibliothek als Lernort eine ganz neue Bedeutung an. Auf lange Sicht ist solch ein Wandel der Organisationskultur eine notwendige Voraussetzung für nachhaltig erfolgreiche neue Bibliotheksdienstleistungen und erfordert ein aktives Change Management. ${ }^{36}$

\section{Ausblick: Alte Werte neu interpretieren}

In diesem Beitrag haben wir ein Konzept für die Vermittlung von Informationskompetenz skizziert, das sich eng am (zunehmend) digitalen Forschungsprozess orientiert. Damit das vorgeschlagene integrierte, genuin bibliothekarische Dienstleistungspaket von den potentiellen Nutzerlnnen auf dem Campus auch tatsächlich dauerhaft angenommen wird, ist neben den oben ausführlich erläuterten Merkmalen, die zur Relevanzperzeption beitragen, ein grundsätzliches Vertrauen in die Bibliothek als Institution erforderlich. Die klassisch der Bibliothek zugeschriebenen Werte der Neutralität, Verlässlichkeit und Qualität bilden eine gute Grundlage, müssen jedoch in der zunehmend digitalen Welt neu interpretiert werden. Wir plädieren hier für eine Rückbesinnung auf die Idee der Offenheit, des freien Zugangs zu Information und Wissen, für die Bibliotheken mit dem

${ }^{30} \mathrm{Vgl}$. Johnson, Larry, Samantha Becker u.a.: NMC Horizon Report. 2015 Higher Education Edition. Austin Texas, [S.I.]: New Media Consortium; EDUCAUSE Learning Initiative 2015. http://www.editlib.org/p/130341/ (7.8.2015).

${ }^{31}$ Vgl. Stephens, Michael: Seek a Challenge | Office Hours. In: Library Journal (2011). http://lj.libraryjournal.com/2011/02/opinion/michael-stephens/seek-a-challenge-office-hours/ (7.8.2015).

32 http://metaliteracy.org/learning-objectives/ (7.8.2015).

${ }^{33}$ Anregungen dazu in Bergmann, Julia: Arbeitsorganisation 2.0. Tools für den Arbeitsalltag in Kultur- und Bildungseinrichtungen. Berlin [u.a.]: DE GRUYTER SAUR 2013 (Praxiswissen). http://dx.doi.org/10.1515/9783110269598 (7.8.2015).

${ }^{34}$ Zur Fort- und Weiterbildung ist beispielsweise das Blog https://bibfobi.wordpress.com einschlägig.

${ }^{35}$ Handbuch CoScience. http://handbuch.io/w/Handbuch_CoScience (7.8.2015).

${ }^{36}$ Erfolgversprechend sind auch hier moderne, partizipative Ansätze, um den Wandel zu gestalten. Vgl. bspw. Appelo, Jürgen: How to Change the World: Change Management 3.0. Jojo Ventures BV 2012. https://management30.com/product/how-to-change-the-world/ (7.8.2015). 
Ziel der Bildung und Selbstaufklärung gegründet wurden. ${ }^{37}$ Eine zeitgemäße Ausgestaltung des alten Wertes der Offenheit wäre beispielsweise eine konsequente Förderung von Open Access, Open Source und Open Science sowie eines Engagements für Netzneutralität und Datenschutz. Ein neues am digitalen Forschungsprozess orientiertes Beratungsangebot wird längerfristig scheitern, wenn nicht gleichzeitig ein Wertewandel erfolgt, der mit einem Wandel der Organisationskultur (wie oben in Bezug auf die Fortbildung beschrieben) eingeleitet werden kann und letztlich integer, d.h. wirklich konsequent und nachhaltig erfolgen muss, um von den KundInnen akzeptiert zu werden. Don Tapscott hat 2012 in einem viel beachteten TED Talk dieses Grundprinzip der "open world" wie folgt beschrieben:

You say you have good products. They'd better be good. But you also need to have values. You need to have integrity as part of your bones and your DNA as an organization, because if you don't, you'll be unable to build trust, and trust is a sine qua non of this new network world. $^{38}$

In Kombination mit konsequent verfolgten, neuinterpretierten Werten wie Offenheit, informationspraktischer Selbstbestimmung oder wissenschaftsethischem Verhalten können die skizzierten Beratungsangebote helfen, der Bibliothek eine zeitgemäße Rolle und Legitimation in der technologiegetriebenen Welt zu geben. Der Bedarf an klassischen Einführungskursen und Schulungen wird durch bessere Benutzbarkeit und Zugänglichkeit von Information und Werkzeugen mittelfristig zurückgehen, die Vermittlung von Methodenwissen wird aber weiterhin benötigt. Wenn wir Bibliothekarinnen und Bibliothekare uns endlich von der Selbstbespiegelung lösen und die Perspektive der NutzerInnen im Forschungsprozess einnehmen, haben wir eine Chance das kulturelle Kapital der Bibliothek in die neue Zeit zu retten.

Das hier skizzierte Konzept ist sicher nur ein Anfang und muss weiter ausgearbeitet und ausdifferenziert werden. Wir würden uns über eine intensive Fachdiskussion sehr freuen und bitten Sie um Ihre Kritik über die Diskussionsplattform Hypothes.is. ${ }^{39}$

\footnotetext{
${ }^{37}$ Lohmeier, Felix u. Jens Mittelbach: Offenheit statt Bündniszwang. In: Zeitschrift für Bibliothekswesen und Bibliographie 61 (2014) H. 4/5. S. 209-215. Preprint verfügbar unter http://nbnresolving.de/urn:nbn:de:bsz:14-qucosa-157772.

${ }^{38}$ Tapscott, Don: Four principles for the open world 2012 (TED Global 2012). http://www.ted.com/talks/don tapscott four principles for the open world 1/transcript?language=en\# $\underline{\mathrm{t}-407180}$ (7.8.2015).

${ }^{39}$ Preprint dieses Beitrags mit Annotationsfunktionen: $\underline{\text { http://slubdd.de/5c. }}$
} 


\section{Literaturverzeichnis}

Appelo, Jürgen: How to Change the World: Change Management 3.0. Jojo Ventures BV 2012. https://management30.com/product/how-to-change-the-world/ (7.8.2015).

Bergmann, Julia: Arbeitsorganisation 2.0. Tools für den Arbeitsalltag in Kultur- und Bildungseinrichtungen. Berlin [u.a.]: DE GRUYTER SAUR 2013 (Praxiswissen). http://dx.doi.org/10.1515/9783110269598 (7.8.2015).

Berstler, Susan, Christopher Erdmann u.a.: Data Management at Harvard. http://isites.harvard.edu/icb/icb.do?keyword=k78759 (7.8.2015).

Bosman, Jeroen u. Bianca Kramer: 101 Innovations in Scholarly Communication. http://innoscholcomm.silk.co (7.8.2015).

DeRosa, Cathy, Joanne Cantrell u.a.: At a tipping point. Education, learning \& libraries; a report to the OCLC membership. Dublin, OH, USA: OCLC 2014. S, 62.

https://www.oclc.org/content/dam/oclc/reports/tipping-point/215133-tipping-point.pdf

Di Rosa, Elena u. Michaela Voigt: Qualitätssicherung für Serviceangebote zur Rechteprüfung für Bibliotheken. Bremen 2014 (103. Deutscher Bibliothekartag). http://nbnresolving.de/urn/resolver.pl?urn:nbn:de:0290-opus-16149 (8.7.2015).

Francese, Enrico: Reference management software as digital libraries. A survey at the University of Torino. Master Thesis. Oslo 2012. http://hdl.handle.net/10642/1274 (7.8.2015)

Guercke, Olaf: Bequem erreichbar und maßgeschneidert, Persönliche Beratungsangebote in der Digitalen Bibliothek. Olaf Guercke und Jens Mittelbach im Gespräch über die Wissensbar der SLUB Dresden. Interview mit Jens Mittelbach (3.8.2015). Erschienen in: b. i. t. online 18 (2015), H. 3. S. 267-268. http://www.b-i-t-online.de/heft/2015-03-interview-guercke.pdf.

Hapke, Thomas: Was unter Informationskompetenz verstanden wird, verändert sich. In HapkeWeblog (21.07.2015) http://blog.hapke.de/information-literacy/was-unterinformationskompetenz-verstanden-wird-veraendert-sich/ (7.8.2015).

Johnson, Larry, Samantha Becker u.a.: NMC Horizon Report. 2015 Higher Education Edition. Austin Texas, [S.I.]: New Media Consortium; EDUCAUSE Learning Initiative 2015. http://www.editlib.org/p/130341/ (7.8.2015).

Lankes, R. David: Beyond the bullet points. Bad libraries build collections, good libraries build services, great libraries build communities (11.3.2012). http://quartz.syr.edu/blog/? $p=1411$ (7.8.2015).

Lemke, Dorothea, Jana Votteler u.a.: Softwarevergleich Literaturverwaltung - 5. Aktualisierung 2015 (30.6.2015). https://mediatum.ub.tum.de/node?id=1271693 (7.8.2015)

Levitin, Daniel J.: The organized mind. Thinking straight in the age of information overload. New York, NY: Dutton 2014.

Lohmeier, Felix u. Jens Mittelbach: Offenheit statt Bündniszwang. In: Zeitschrift für Bibliothekswesen und Bibliographie 61 (2014) H. 4/5. S. 209-215. Preprint verfügbar unter http://nbnresolving.de/urn:nbn:de:bsz:14-qucosa-157772.

Mackey, Thomas P. u. Trudi E. Jacobson: Metaliteracy. Reinventing information literacy to empower learners. Chicago: ALA Neal-Schuman 2014.

McMinn, Howard Stephen: Library support of bibliographic management tools. A review. In:

Reference Services Review 39 (2011) H. 2. S. 278-302.

http://dx.doi.org/10.1108/00907321111135493 (7.8.2015) 
Mittelbach, Jens u. Antonie Muschalek: Wissensbar. Experten beraten passgenau. In: BuB: Forum Bibliothek und Information 67 (2015) H. 6. S. 374-376. http://b-u-b.de/wpcontent/uploads/2015-06.pdf.

Musiat, Jutta, Annika-Valeska Walzel u. Christina Wenzel: Literaturverwaltungsprogramme im Überblick (Januar 2015). http://slubdd.de/5b (7.8.2015).

Nagelschmidt, Matthias: Literaturverwaltungsprogramme. Das funktionale Minimum. In: ABI-Technik 30 (2010) H. 2. http://dx.doi.org/10.1515/ABITECH.2010.30.2.94 (07.08.2015)

Shank, John D., Steven Bell u. Diane Zabel: Blended librarianship. [Re]envisioning the role of librarian as educator in the digital information age. In: Reference and User Services Quarterly 51 (2011) H. 2. S. 105-110. http://www.jstor.org/stable/refuseserq.51.2.105 (7.8.2015)

Siegfried, Doreen: Wissenschaftsservice der Zukunft. ZBW definiert Forschungssupport neu. Pressemitteilung der ZBW (7.12.2010). http://www.zbw.eu/de/ueberuns/aktuelles/meldung/news/wissenschaftsservice-der-zukunft-zbw-definiertforschungssupport-neu/ (7.8.2015).

Stephens, Michael: Seek a Challenge | Office Hours. In: Library Journal (2011). http://lj.libraryjournal.com/2011/02/opinion/michael-stephens/seek-a-challenge-office-hours/ (7.8.2015).

Stöber, Thomas: Serviceangebote der wissenschaftlichen Bibliotheken im Bereich Literaturverwaltung: Ergebnisse einer Umfrage. Augsburg 2010. http://opus.bibliothek.uniaugsburg.de/volltexte/2010/1611 (7.8.2015)

Stöber, Thomas: Informationskompetenz und Literaturverwaltung. In: Handbuch Informationskompetenz. Hrsg. von Wilfried Sühl-Strohmenger. 1. Aufl. Berlin [u.a.]: De Gruyter 2012. http://dx.doi.org/10.1515/9783110255188.281 (07.08.2015)

Stöber, Thomas: Der "digitale Schreibtisch". Innovationsmanagement und Vernetzung für das wissenschaftliche Arbeiten. In: Literaturwissenschaft im digitalen Medienwandel. Hrsg. von Christof Schöch u. Lars Schneider. Berlin 2014 (PhiN. Philologie im Netz: Beihefte). S. 180-188. http://userpage.fu-berlin.de/ phin/beiheft7/b7t11.pdf (7.8.2015).

Stöhr, Matti: Bibliothekarische Dienstleistungen für Literaturverwaltung. Eine vergleichende Analyse des Angebots wissenschaftlicher Bibliotheken unter besonderer Berücksichtigung der Nutzerperspektive. Magisterarbeit. Berlin 2010. http://nbn-resolving.de/urn:nbn:de:kobv:11-100178393 (07.08.2015)

Stöhr, Matti: Neuer Titel, neue Möglichkeiten - "Literaturverwaltung \& Bibliotheken” heißt nun "Literaturverwaltung". In: Literaturverwaltung (10.05.2013). https://literaturverwaltung.wordpress.com/2013/05/10/neuer-titel-neue-moglichkeitenliteraturverwaltung-bibliotheken-heist-nun-literaturverwaltung/ (7.8.2015)

Süselbeck, Kirsten: Literaturverwaltung als Teil des Konzepts zur Vermittlung von Informationskompetenz an wissenschaftlichen Bibliotheken 2011. http://bvbm1.bibbvb.de/publish/viewer/16/2805073.html (7.8.2015)

Tappenbeck, Inka: Fachreferat 2020. ZBW Hamburg 2014 (VDB: Fachreferententagung Wirtschaftswissenschaften). http://www.fbi.fhkoeln.de/institut/personen/tappenbeck/publikationen/Fachreferat 2020 Praesentation.pdf (7.8.2015).

Tapscott, Don: Four principles for the open world 2012 (TED Global 2012). http://www.ted.com/talks/don tapscott four principles for the open world 1/transcript?lang uage=en\#t-407180 (7.8.2015). 


\section{Metadaten}

Schlüsselbegriffe: Informationskompetenz, Openness, Forschungszyklus, Forschungsprozess, Dienstleistung, Research Management, Community Building, Literaturverwaltung, Blended Librarian, Metaliteracy

\section{Kurzbiografie Lohmeier:}

Felix Lohmeier leitet seit 2013 die IT-Abteilung der Sächsischen Landesbibliothek - Staats- und Universitätsbibliothek Dresden (SLUB). Zuvor war er als Leiter des Referats Informationsservice für den Ausbau des Bereichs Elektronisches Publizieren zuständig. Neben der technischen Interoperabilität von Bibliothekssystemen gilt sein Interesse auch dem freien Zugang zu wissenschaftlicher Literatur und Daten.

http://felixlohmeier.de

\section{Kurzbiografie Mittelbach:}

Dr. Jens Mittelbach ist seit 2010 Leiter der Abteilung Benutzung und Information der SLUB Dresden. Zuvor war er an der SUB Göttingen Fachreferent. Er studierte Anglistik und Germanistik, promovierte im Fach Englische Literatur und absolvierte ein postgraduales Studium der Bibliotheks- und Informationswissenschaft im Rahmen seines Bibliotheksreferendariats an der UB der FU Berlin. Seine Interessen gelten den Themen Openness und Open Data, Informationstechnologie und Datenmanagement sowie Bibliotheksservices der Zukunft.

http://jensmittelbach.de

\section{Kurzbiografie Stöhr:}

Matti Stöhr ist hauptberuflich seit Februar 2011 als wissenschaftlich-technischer, vorrangig als wissenschaftlicher (Projekt-)Mitarbeiter im Akademienvorhaben "Jahresberichte für deutsche Geschichte" tätig - einer an der Berlin-Brandenburgischen Akademie der Wissenschaften gepflegten fachbibliographischen Datenbank. Er ist zudem unter anderem Mitbegründer und Redakteur der Webplattform "Literaturverwaltung". Seine Interessen gelten insbesondere den Themen Literaturverwaltung/Research Management, bibliotheks-/informationswissenschaftliche und historische Fachkommunikation sowie Informationsservices für die Wissenschaft im Allgemeinen.

http://matti-stoehr.de 